\title{
Electronic Coherence in Ultrafast X-Ray Scattering from Molecular Wave Packets
}

Simmermacher, Mats; Henriksen, Niels E.; Møller, Klaus B.; Moreno Carrascosa, Andrés; Kirrander, Adam

Published in:

Physical Review Letters

Link to article, DOI:

10.1103/PhysRevLett.122.073003

Publication date:

2019

Document Version

Publisher's PDF, also known as Version of record

Link back to DTU Orbit

Citation (APA):

Simmermacher, M., Henriksen, N. E., Møller, K. B., Moreno Carrascosa, A., \& Kirrander, A. (2019). Electronic Coherence in Ultrafast X-Ray Scattering from Molecular Wave Packets. Physical Review Letters, 122(7), [073003]. https://doi.org/10.1103/PhysRevLett.122.073003

\section{General rights}

Copyright and moral rights for the publications made accessible in the public portal are retained by the authors and/or other copyright owners and it is a condition of accessing publications that users recognise and abide by the legal requirements associated with these rights.

- Users may download and print one copy of any publication from the public portal for the purpose of private study or research.

- You may not further distribute the material or use it for any profit-making activity or commercial gain

- You may freely distribute the URL identifying the publication in the public portal 


\title{
Electronic Coherence in Ultrafast X-Ray Scattering from Molecular Wave Packets
}

\author{
Mats Simmermacher, Niels E. Henriksen, and Klaus B. Møller ${ }^{*}$ \\ Department of Chemistry, Technical University of Denmark, 2800 Lyngby, Denmark \\ Andrés Moreno Carrascosa and Adam Kirrander ${ }^{\dagger}$ \\ EaStCHEM, School of Chemistry, University of Edinburgh, EH9 3FJ Edinburgh, United Kingdom
}

(Received 31 August 2018; published 22 February 2019)

\begin{abstract}
Simulations of nonresonant ultrafast $\mathrm{x}$-ray scattering from a molecular wave packet in $\mathrm{H}_{2}$ are used to examine and classify the components that contribute to the total scattering signal. The elastic component, which can be used to determine the structural dynamics of the molecule, is also found to carry a strong signature of an adiabatic electron transfer that occurs in the simulated molecule. The inelastic component, frequently assumed to be constant, is found to change with the geometry of the molecule. Finally, a coherent mixed component due to interferences between different inelastic transitions is identified and shown to provide a direct probe of transient electronic coherences.
\end{abstract}

DOI: 10.1103/PhysRevLett.122.073003

$\mathrm{X}$-ray scattering plays a critical role in the determination of the structure of matter [1], and its extension into the temporal domain opens a window onto the structural dynamics of chemical and physical transformations [2-4]. The emergence of $x$-ray free-electron lasers (XFELs) [5-7] means that ultrafast $\mathrm{X}$-ray scattering now rivals the timeresolution of ultrafast spectroscopies and can probe the structural dynamics of fundamental chemical events such as the breaking of bonds, molecular vibrations, isomerization, and solvation [8-14]. However, photochemical and photophysical processes are governed by a complex interplay of nuclear and electronic motion, and structural dynamics alone does not tell the full story $[15,16]$.

The opportunity for $\mathrm{x}$-ray scattering experiments that exploit the short duration and coherence of XFEL pulses to reach beyond structural dynamics [17-20] is explored in this Letter. Via accurate simulations of scattering from a molecular wave packet, we identify three distinct contributions to the total scattering signal: elastic, inelastic, and coherent mixed. The coherent mixed component is qualitatively different from the other two. It relies on interferences between different coherently populated electronic states and displays the same distinctive features as the scattering predicted for electronic wave packets in atoms [21-23]. In a molecule, these features are found to provide a direct signature of transient electronic coherences and could be exploited to characterize the evolution of molecular wave packets at avoided crossings, conical intersections, or during partial revivals. This Letter provides the first predictions of how this type of signal might appear on the detector in an experiment. In the course of making this detailed analysis, important aspects of the elastic and inelastic scattering are uncovered.

Ultrafast nonresonant $\mathrm{x}$-ray scattering is described by first-order perturbation theory and in terms of a fully quantized description of the $\mathrm{x}$-ray pulse [21,24-27]. The time-resolved differential x-ray scattering signal $d \sigma / d \Omega$ per solid angle $\Omega$ for a general material system is, then, given by

$$
\begin{aligned}
\frac{d \sigma}{d \Omega}= & \left(\frac{d \sigma}{d \Omega}\right)_{\mathrm{Th}} \iiint_{\frac{\omega_{\mathrm{s}}}{\omega_{0}} I(t) C(\delta) e^{l\left(\omega_{0}-\omega_{\mathrm{s}}\right) \delta}} \\
& \times \mathcal{L}(\boldsymbol{q}, t, \delta) d \delta d \omega_{\mathrm{s}} d t,
\end{aligned}
$$

where $(d \sigma / d \Omega)_{\mathrm{Th}}$ is the differential Thomson scattering cross section of the free electron, $I(t)$ the photon number intensity, and $C(\delta)$ the linear coherence function of the $\mathrm{x}-$ ray probe pulse with their corresponding times $t$ and $\delta$, and $\omega_{0}$ and $\omega_{\mathrm{s}}$ are the angular frequencies of incident and scattered photons. The quantity $\mathcal{L}(\boldsymbol{q}, t, \delta)$ defines the scattering probability and is analogous to the dynamic structure factor $S\left(\boldsymbol{q}, \omega_{0}-\omega_{\mathrm{s}}\right)$ [28]. It is expressed as

$\mathcal{L}(\boldsymbol{q}, t, \delta)=\left\langle\Psi(t)\left|e^{l \hat{H}_{M} \delta / 2 \hbar} \hat{L}^{\dagger} e^{-l \hat{H}_{M} \delta / \hbar} \hat{L} e^{l \hat{H}_{M} \delta / 2 \hbar}\right| \Psi(t)\right\rangle$,

where $\hbar=h / 2 \pi$ is Planck's constant, $\iota$ the imaginary unit, and the bracket implies integration over all electronic $\overline{\boldsymbol{r}}=\left(\boldsymbol{r}_{1}, \ldots, \boldsymbol{r}_{N_{\mathrm{e}}}\right)$ and nuclear $\overline{\boldsymbol{R}}=\left(\boldsymbol{R}_{1}, \ldots, \boldsymbol{R}_{N_{\mathrm{at}}}\right)$ coordinates. Furthermore, Eq. (2) contains the time-dependent wave function $|\Psi(t)\rangle$, the field-free molecular Hamiltonian $\hat{H}_{M}$, and the electronic scattering operator $\hat{L}=\sum_{n} e^{q r_{n}}$, with the scattering vector $\boldsymbol{q}=\boldsymbol{k}_{0}-\boldsymbol{k}_{\mathrm{s}}$ taken as the difference between the wave vectors of the incident and scattered photons.

The drawback of Eq. (1) is that it is unwieldy for actual calculations and provides little physical insight into the scattering process. We therefore introduce an explicit Born-Huang form for the molecular wave function 
$\langle\overline{\boldsymbol{r}}, \overline{\boldsymbol{R}} \mid \Psi(t)\rangle=\Psi(\overline{\boldsymbol{r}}, \overline{\boldsymbol{R}}, t)=\sum_{i}^{N} \chi_{i}(\overline{\boldsymbol{R}}, t) \varphi_{i}(\overline{\boldsymbol{r}} ; \overline{\boldsymbol{R}})$ expressed in a direct-product basis of electronic eigenfunctions $\varphi_{i}(\overline{\boldsymbol{r}} ; \overline{\boldsymbol{R}})$ and nuclear wave packets $\chi_{i}(\overline{\boldsymbol{R}}, t)$. Furthermore, we assume that scattering involving rovibrational transitions is not resolved. This allows us to expand Eq. (2) using the resolution of identity for electronic states, $\hat{\mathbf{1}}=$ $\sum_{f}\left|\varphi_{f}(\overline{\boldsymbol{R}})\right\rangle\left\langle\varphi_{f}(\overline{\boldsymbol{R}})\right|$, and to evaluate the effect of the molecular Hamiltonian on the electronic eigenstates as $e^{-l \hat{H}_{M} \delta / \hbar} \varphi_{i}(\overline{\boldsymbol{r}} ; \overline{\boldsymbol{R}}) \approx e^{-l V_{i}(\overline{\boldsymbol{R}}) \delta / \hbar} \varphi_{i}(\overline{\boldsymbol{r}} ; \overline{\boldsymbol{R}})$ with $V_{i}(\overline{\boldsymbol{R}})$ the eigenvalues of the electronic Hamiltonian. These assumptions yield

$$
\begin{aligned}
\mathcal{L}(\boldsymbol{q}, t, \delta) \approx & \sum_{i, j}^{N} \sum_{f}^{\infty} e^{l \omega_{f i j} \delta} \int \chi_{i}(\overline{\boldsymbol{R}}, t) \chi_{j}^{*}(\overline{\boldsymbol{R}}, t) L_{f i}(\boldsymbol{q}, \overline{\boldsymbol{R}}) \\
& \times L_{f j}^{*}(\boldsymbol{q}, \overline{\boldsymbol{R}}) d \overline{\boldsymbol{R}}
\end{aligned}
$$

where $L_{f i}(\boldsymbol{q}, \overline{\boldsymbol{R}})=\left\langle\varphi_{f}(\overline{\boldsymbol{R}})|\hat{L}| \varphi_{i}(\overline{\boldsymbol{R}})\right\rangle=\left\langle\varphi_{i}(\overline{\boldsymbol{R}})\left|\hat{L}^{\dagger}\right| \varphi_{f}(\overline{\boldsymbol{R}})\right\rangle^{*}$ and $\omega_{f i j}=\left(V_{f}-\left[V_{i}+V_{j}\right] / 2\right) / \hbar$ with the electronic eigenvalues evaluated at equilibrium geometry, e.g., $V_{i}=V_{i}\left(\overline{\boldsymbol{R}}_{0}\right)$.

A final simplification is achieved if we recognize that the difference in energy of the incident and the scattered photons is small in comparison to the mean photon energy of the x-ray pulse, $\omega_{\mathrm{s}} \approx \omega_{0}$, which renders the $\boldsymbol{q}$ vector independent of $\omega_{\mathrm{S}}$ [29] (indicated by $\tilde{\boldsymbol{q}}$, henceforth). This allows us to simplify the integrals over $\omega_{\mathrm{s}}$ and $\delta$ in Eq. (1) to obtain a window function $W_{f i j}(\Delta \omega)$ (see Supplemental Material (SM) [30]). The detection window $\Delta \omega$ defines the range of detected photons around the mean $\omega_{0}$.

Combining these results, we arrive at a compact and computationally practical expression which offers significant physical insight

$$
\begin{aligned}
\frac{d \sigma}{d \boldsymbol{\Omega}} \approx & \left(\frac{d \sigma}{d \boldsymbol{\Omega}}\right) \sum_{\mathrm{Th}} \sum_{i, j}^{N} \sum_{f}^{\infty} W_{f i j}(\Delta \omega) \int I(t) \int \chi_{i}(\overline{\boldsymbol{R}}, t) \chi_{j}^{*}(\overline{\boldsymbol{R}}, t) \\
& \times L_{f i}(\tilde{\boldsymbol{q}}, \overline{\boldsymbol{R}}) L_{f j}^{*}(\tilde{\boldsymbol{q}}, \overline{\boldsymbol{R}}) d \overline{\boldsymbol{R}} d t .
\end{aligned}
$$

Three contributions to the x-ray scattering signal can be identified in Eq. (4): elastic $(i=j=f)$, inelastic $(i=j \neq f)$, and coherent mixed $(i \neq j$, any $f)$. The same partitioning was previously used by Cao and Wilson [17] and by Mukamel et al. [18,19]. The elastic and inelastic components are related to the probability density of the nuclear wave packet on each electronic state $\left|\chi_{i}(\overline{\boldsymbol{R}}, t)\right|^{2}$ and to the absolute square of the scattering matrix elements $\left|L_{f i}(\tilde{\boldsymbol{q}}, \overline{\boldsymbol{R}})\right|^{2}$. The inelastic matrix elements are smaller than the elastic, but become significant when summed and cannot be generally neglected. The coherent mixed component, in turn, is an interference effect with no correspondence in standard $\mathrm{x}$-ray scattering. It is due to inelastic transitions from the occupied states $i$ and $j$ to a third state $f$, weighted by the nuclear wave packet overlap density $\chi_{i}(\overline{\boldsymbol{R}}, t) \chi_{j}^{*}(\overline{\boldsymbol{R}}, t)$. The strongest contribution comes from

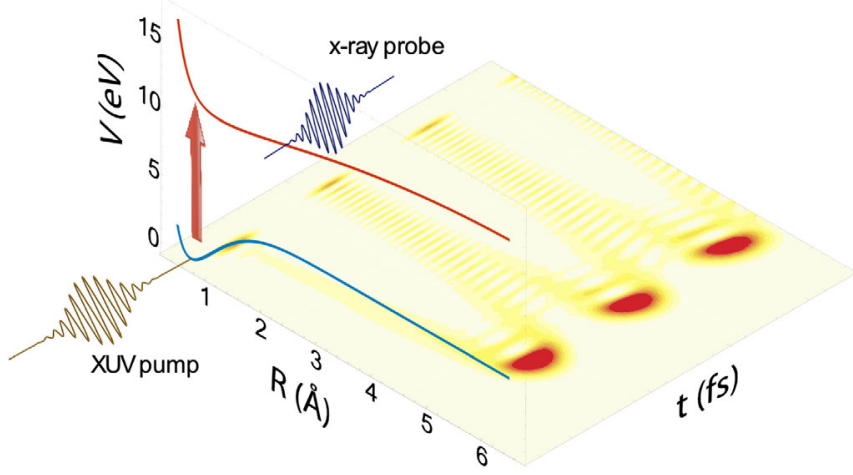

FIG. 1. Schematic of the proposed experiment. An extreme ultraviolet (XUV) pump pulse excites a wave packet from the $X^{1} \Sigma_{g}^{+}$ground state of $\mathrm{H}_{2}$ (blue curve) onto the $B^{1} \Sigma_{u}^{+}$state (red curve). The ground state nuclear wave packet remains stationary, while the $B$-state wave packet oscillates between the inner and outer turning points as shown in the contour plot of the probability density $\left|\chi_{B}(R, t)\right|^{2}$ as a function of internuclear distance $R(\AA)$ and time $t$ (fs). The potential energy curves $V$ $(\mathrm{eV})$ of the two states are shown. After excitation, an x-ray pulse probes the system by nonresonant ultrafast scattering.

terms with $f=i$ or $f=j$, where one of the matrix elements is elastic and, thus, large. These interferences, mediated by the inelastic scattering matrix elements, are intramolecular in a noncrystalline sample [18-20,46].

We explore the behavior implied by Eq. (4) in realistic simulations of $\mathrm{H}_{2}$, a benchmark molecule [47] whose stationary elastic and inelastic x-ray scattering has been measured recently $[48,49]$. A schematic outline of the simulations is shown in Fig. 1 with details provided in the SM [30]. The time-evolution of the molecule is calculated by numerically integrating the time-dependent Schrödinger equation with the WAVEPACKET code [31] using accurate potential energies, dipole transition moments, and masses [33-36]. A $14.3 \mathrm{eV}$ transform limited pump pulse with $25 \mathrm{fs}$ duration (FWHM) centered at time $t=0 \mathrm{fs}$ with peak intensity $7.69 \times 10^{12} \mathrm{~W} / \mathrm{cm}^{2}$ is included explicitly and excites a $B^{1} \Sigma_{u}^{+} \leftarrow X^{1} \Sigma_{g}^{+}(\nu=0)$ wave packet with $10 \%$ population transfer from the ground state. A similar wave packet has been observed experimentally [50]. While the ground state is simply depleted by the pump pulse and remains essentially stationary, the nuclear component on the electronic $B$ state oscillates between the inner and outer turning points with a vibrational period of $T_{\mathrm{vib}} \approx 62 \mathrm{fs}$.

To determine the scattering signal by means of Eq. (4), the elastic and inelastic scattering matrix elements $L_{f i}(\tilde{\boldsymbol{q}}, R)$ are calculated among the nine lowest-energy electronic singlet states using our own code [41,42]. The electronic wave functions are obtained by state-averaged CASSCF $(2,30) / d$-aug-cc-pVQZ in MOLPRO [40] (see SM [30]). The X-ray pulse is taken to be coherent and transform limited with duration $d_{\mathrm{x}}=0.1 \mathrm{fs}$ (FWHM), its mean photon energy $\hbar \omega_{0}=8.5 \mathrm{keV}$, and a detection window of $\hbar \Delta \omega=50.0 \mathrm{eV}$ is assumed. The molecule is aligned 


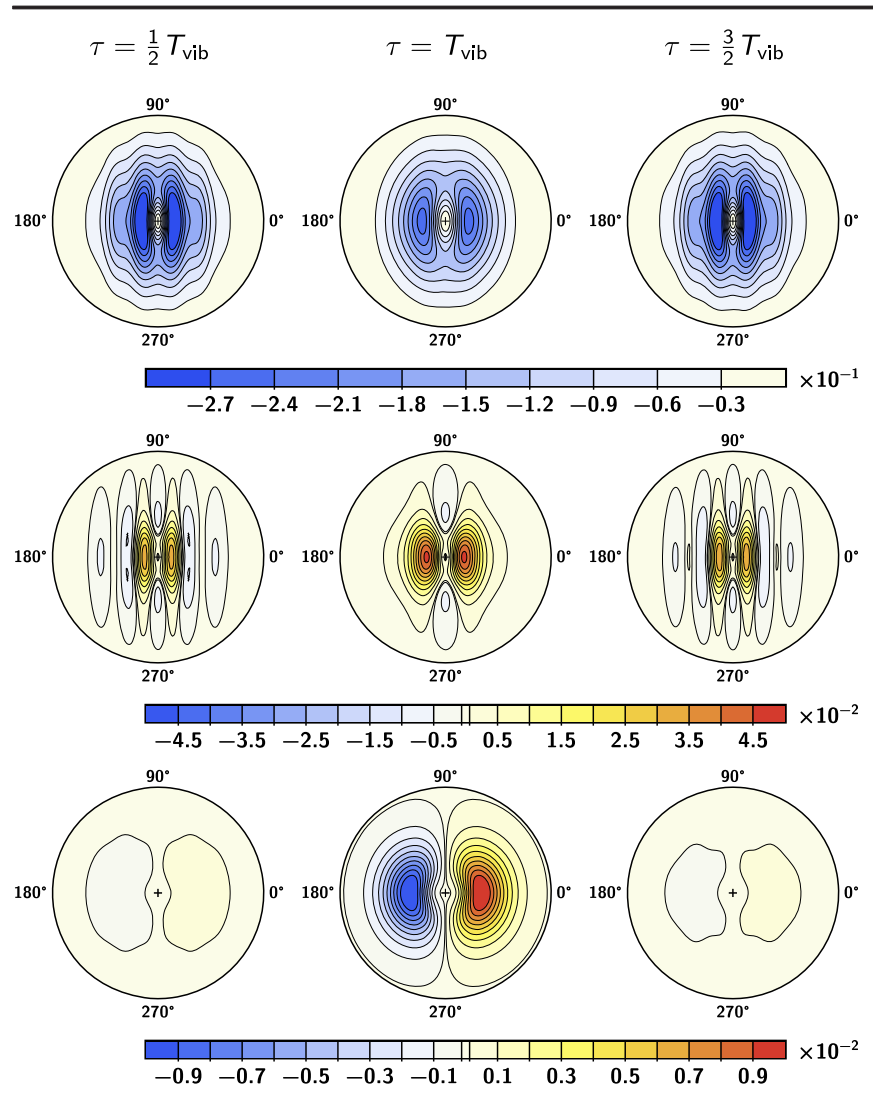

FIG. 2. Detector images showing the difference $x$-ray scattering patterns (pump on - pump off) for the elastic (top row), inelastic (middle row), and coherent mixed (bottom row) components of the scattering signal at pump-probe delays $\frac{1}{2} T_{\text {vib }}, T_{\text {vib }}$, and $\frac{3}{2} T_{\text {vib }}$. The scattering intensity is given in units of the Thomson scattering cross section and the norm of the scattering vector is $0 \leq q \leq 4.31 \AA^{-1}$.

with the laboratory $\hat{x}$ axis and the incident $\mathrm{x}$-ray pulse propagates along the laboratory $\hat{z}$ axis, while scattering images are calculated in the $\left(q_{x}, q_{y}\right)$ plane. Difference (pump on - pump off) images of the elastic, inelastic, and coherent mixed components of the scattering signal are shown in Fig. 2 at delay-times $\frac{1}{2} T_{\text {vib }}, T_{\text {vib }}$, and $\frac{3}{2} T_{\text {vib }}$ corresponding to the outer, inner, and outer turning points of the wave packet. Now, we will discuss each of the contributions in turn.

The elastic component is shown in the top row of Fig. 2. This contribution is the largest, especially at small scattering angles, and the images are centrosymmetric as expected from Friedel's law [51]. Importantly, the signal relates to the Fourier transform of the electron density and can be inverted to provide the spatiotemporal structural dynamics of the molecule [26,52]. The images are negative at all pump-probe delays, reflecting that the electron density of the excited molecule is more diffuse than for the unpumped ground-state molecule. The time-dependent changes in the elastic pattern reflect the changes in the electron density as the nuclear wave packet on the electronic $B$ state oscillates between the inner and outer turning points. Isosurfaces for
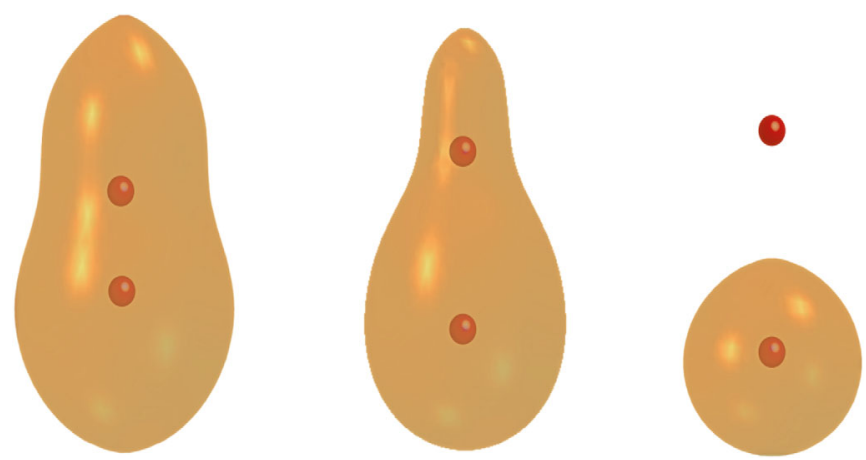

FIG. 3. Isosurfaces of the net electron density (see SM [30]) associated with the $B$-state component of the molecular wave packet at times $T_{\text {vib }}, \frac{5}{4} T_{\text {vib }}$, and $\frac{6}{4} T_{\text {vib }}$ (left to right). The mean positions of the nuclei are indicated by red circles. The expectation value of the internuclear distance increases from $\langle R\rangle \approx$ $2.3 \AA$ at the inner turning point, via $\langle R\rangle \approx 4.1 \AA$, to $\langle R\rangle \approx 5.1 \AA$ at the outer turning point (left to right). Note that the molecule has been rotated by $90^{\circ}$ with respect to Figs. 2 and 5 .

the net electron density associated with the $B$-state component of the wave packet are shown in Fig. 3 at three different times that correspond to increasing internuclear separation. It is notable that the electron density is more compact at large internuclear distances due to adiabatic electron transfer resulting in a strong ion-pair character $H^{+} H^{-}\left(1 s^{2}\right)$ and a marked deviation from the common independent atom model [45].

The inelastic component shown in the middle row of Fig. 2 displays the same centrosymmetry as the elastic pattern but is weaker overall. The values of the inelastic difference signal are mainly positive, reflecting that inelastic transitions from the $B$ state are more likely than from the ground state. The most interesting observation is that the inelastic signal changes significantly in the course of the dynamics. This is due to the fact that the inelastic scattering matrix elements change as a function of the internuclear distance. In $\mathrm{H}_{2}$, the magnitude of the inelastic scattering matrix elements is largest close to the equilibrium distances of the corresponding electronic states.

The coherent mixed component is shown in the bottom row of Fig. 2. It does not share the centrosymmetry of the elastic and inelastic components but undergoes a sign change when reflected through the vertical $q_{y}$ axis. This could be exploited to isolate the coherent mixed component from the total scattering using Legendre polynomial decomposition. At its peak, the coherent mixed component has the same order of magnitude as the inelastic contribution. It undergoes a modulation with period $T_{\text {vib }} \approx 62 \mathrm{fs}$ that causes the images at delay times $\frac{1}{2} T_{\text {vib }}$ and $\frac{3}{2} T_{\text {vib }}$ in Fig. 2 to be quite faint. This can be seen more clearly by inspection of the integrated absolute value of the coherent mixed component of the scattering signal $\Upsilon(\tau)$ shown in Fig. 4.

The observed modulation can be traced to the overlap density factor $\chi_{B}(R, t) \chi_{X}^{*}(R, t)$ in Eq. (4) and is analogous 


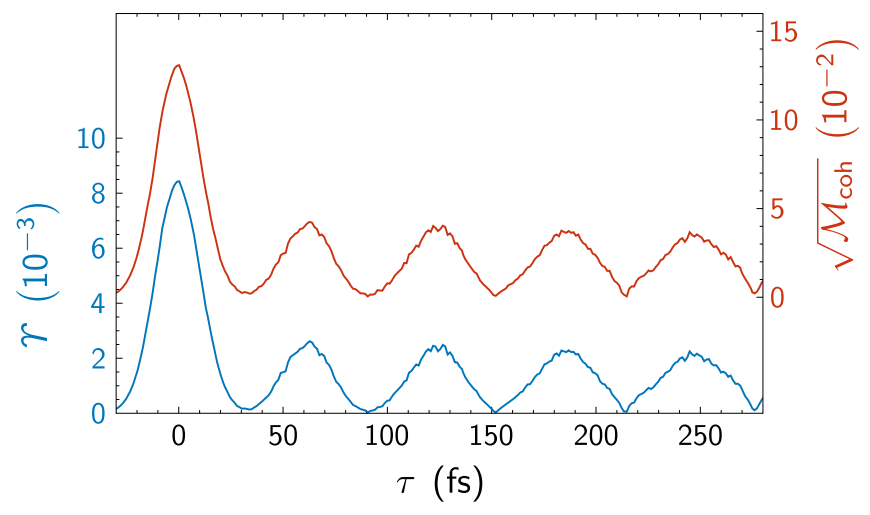

FIG. 4. (Top, right axis) Square root of the coherence function $\mathcal{M}_{\text {coh }}(\tau)$ at different pump-probe delays $\tau$ calculated from the simulated ground and excited state wave packets of the $\mathrm{H}_{2}$ molecule. (Bottom, left axis) Envelope of the integrated absolute value of the coherent mixed component of the scattering signal $\Upsilon(\tau)$.

to transient electronic coherences in molecules ionized by short duration broadband pulses [43,53]. The degree of coherence can be characterized by $\mathcal{M}_{\mathrm{coh}}(\tau)=$ $\operatorname{Tr}\left[\boldsymbol{\rho}_{\text {red }}^{2}(\tau)\right]-\sum_{i}\left(\rho_{\text {red }}^{i i}(\tau)\right)^{2}$, where $\rho_{\text {red }}^{i j}(\tau)=\left\langle\chi_{j}(\tau) \mid \chi_{i}(\tau)\right\rangle$ are elements of the reduced density matrix (see SM [30]) [44]. The values of the square root of $\mathcal{M}_{\mathrm{coh}}(\tau)$ for the current simulations are shown in the top of Fig. 4. They map perfectly onto $\Upsilon(\tau)$ in the bottom of Fig. 4. It is worth noting that the decrease of the maxima in the envelope of the integrated coherent mixed scattering signal provides quantitative information about the dispersion of the nuclear wave packet.

Further insight into the nature of the coherent mixed component is hinted at by the rapid oscillations observed under the slowly modulating envelope, as shown in Fig. 5. The period of these oscillations is $T_{\text {elec }} \approx 0.3$ fs and matches the pulse energy that excites the molecule from the ground to the excited state. The beating is due to interference between the two electronic states and is also visible in the electron density, reflected by the transition densities of the $X$ and $B$ states displayed in Fig. 5. The temporal phase shift between the observed coherent mixed scattering and the transition densities is remarkably similar to what has been observed for electronic wave packets in the hydrogen atom [21,23].

We should note that the present simulations are as close to numerical convergence as practically possible, but the strengths of the inelastic and the coherent mixed components relative to the elastic signal are somewhat underestimated. Qualitatively, however, the simulation provides correct results. The symmetries of the patterns, their temporal evolution, and the trends in their changes become manifest. We also note that the current example is not ideally chosen from the experimental point of view. The $14.3 \mathrm{eV}$ gap between the two electronic states causes rapid oscillations in the coherent mixed part that require subfemtosecond pulses to be resolved [54]. Moreover, detection of all transitions within the molecule would cause the coherence

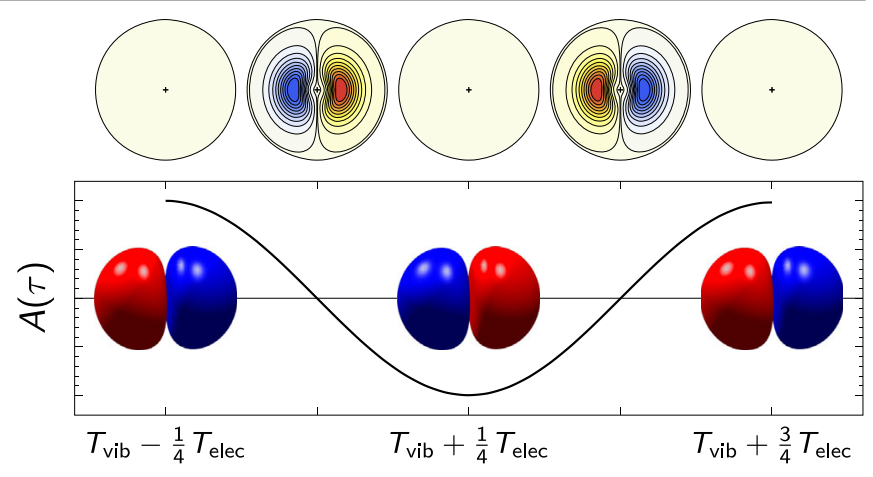

FIG. 5. (Top) The coherent mixed part of the scattering signal, as in Fig. 2, at pump-probe delays $T_{\text {vib }}-\frac{1}{4} T_{\text {elec }}, T_{\text {vib }}$, $T_{\text {vib }}+\frac{1}{4} T_{\text {elec }}, T_{\text {vib }}+\frac{2}{4} T_{\text {elec }}$, and $T_{\text {vib }}+\frac{3}{4} T_{\text {elec }}$ (left to right). (Bottom) Illustration of the one-electron transition density between the $X^{1} \Sigma_{g}^{+}$and $B^{1} \Sigma_{u}^{+}$states and the associated value of the overlap $A(\tau)=\operatorname{Re}\left[\left\langle\chi_{X}(\tau) \mid \chi_{B}(\tau)\right\rangle\right]$.

mixed component of the scattering signal to vanish (see SM [30]), and therefore, it is necessary to ensure that only photons that excite to the eigenstates with the lowest energies are detected with significant weight. Finally, at least partial alignment is necessary to observe the coherent mixed component and its symmetry-breaking property.

To conclude, our results emphasize the importance of an appropriate theoretical framework for the interpretation and design of new ultrafast scattering experiments $[19,21,55]$. The description embodied by Eq. (4) is suitable for quantum molecular dynamics simulations and should aid the disentangling of the different components to the total scattering. Importantly, our calculations of the elastic and inelastic scattering highlight failures of the independent atom model commonly used to interpret structural dynamics, while the observed coherent mixed component points towards new ultrafast $\mathrm{x}$-ray scattering experiments capable of providing valuable information beyond structural dynamics. In the current example, it provides a measure of the dispersion of the nuclear wave packet at partial revivals, and could be used to probe transient coherences at conical intersections known to play a critical role in photochemistry and photophysics. In general, the coherent mixed scattering gives direct access to the degree of electronic coherence and associated electron dynamics and could grow into an important tool for probing coherence in a wide range of systems.

A. K. acknowledges support from a Royal Society of Edinburgh Sabbatical Fellowship (Grant No. 58507) and, with A. M. C., a research grant from the Carnegie Trust for the Universities of Scotland (Grant No. CRG050414). M. S. acknowledges support from HPC-EUROPA3 (Grant No. INFRAIA-2016-1-730897). The computational work reported used the ARCHER UK National Supercomputing Service ([56]) and support from the Edinburgh Parallel Computing Center (EPCC) is acknowledged.

M. S. and A. M. C. have contributed equally to this work. 
*kbmo@kemi.dtu.dk

†adam.kirrander@ed.ac.uk

[1] J. Als-Nielsen and D. McMorrow, Elements of Modern $X$-Ray Physics, 2nd ed. (Wiley-Blackwell, Chichester, 2011).

[2] J. P. Bergsma, M. H. Coladonato, P. M. Edelsten, J. D. Kahn, K. R. Wilson, and D. R. Fredkin, J. Chem. Phys. 84, 6151 (1986).

[3] R. Neutze, R. Wouts, S. Techert, J. Davidsson, M. Kocsis, A. Kirrander, F. Schotte, and M. Wulff, Phys. Rev. Lett. 87, 195508 (2001).

[4] H. Ihee, M. Lorenc, T. K. Kim, Q. Y. Kong, M. Cammarata, J. H. Lee, S. Bratos, and M. Wulff, Science 309, 1223 (2005).

[5] C. Bostedt et al., J. Phys. B 46, 164003 (2013).

[6] J. Feldhaus, M. Krikunova, M. Meyer, T. Möller, R. Moshammer, A. Rudenko, T. Tschentscher, and J. Ullrich, J. Phys. B 46, 164002 (2013).

[7] M. Yabashi, H. Tanaka, T. Tanaka, H. Tomizawa, T. Togashi, M. Nagasono, T. Ishikawa, J. R. Harries, Y. Hikosaka, A. Hishikawa, K. Nagaya, N. Saito, E. Shigemasa, K. Yamanouchi, and K. Ueda, J. Phys. B 46, 164001 (2013).

[8] K. H. Kim et al., Nature (London) 518, 385 (2015).

[9] M. Levantino, G. Schiró, H. T. Lemke, G. Cottone, J. M. Glownia, D. Zhu, M. Chollet, H. Ihee, A. Cupane, and M. Cammarata, Nat. Commun. 6, 6772 (2015).

[10] M. P. Minitti, J. M. Budarz, A. Kirrander, J. Robinson, T. J. Lane, D. Ratner, K. Saita, T. Northey, B. Stankus, V. CoferShabica, J. Hastings, and P. M. Weber, Faraday Discuss. 171, 81 (2014).

[11] M. P. Minitti, J. M. Budarz, A. Kirrander, J. S. Robinson, D. Ratner, T. J. Lane, D. Zhu, J. M. Glownia, M. Kozina, H. T. Lemke, M. Sikorski, Y. Feng, S. Nelson, K. Saita, B. Stankus, T. Northey, J. B. Hastings, and P. M. Weber, Phys. Rev. Lett. 114, 255501 (2015).

[12] B. Stankus, J. M. Budarz, A. Kirrander, D. Rogers, J. Robinson, T. J. Lane, D. Ratner, J. Hastings, M. P. Minitti, and P. M. Weber, Faraday Discuss. 194, 525 (2016).

[13] E. Biasin et al., Phys. Rev. Lett. 117, 013002 (2016).

[14] T. B. van Driel et al., Nat. Commun. 7, 13678 (2016).

[15] A. Stolow, Faraday Discuss. 163, 9 (2013).

[16] A. D. Smith, E. M. Warne, D. Bellshaw, D. A. Horke, M. Tudorovskya, E. Springate, A. J. H. Jones, C. Cacho, R. T. Chapman, A. Kirrander, and R. S. Minns, Phys. Rev. Lett. 120, 183003 (2018).

[17] J. Cao and K. R. Wilson, J. Phys. Chem. A 102, 9523 (1998).

[18] M. Kowalewski, K. Bennett, and S. Mukamel, Struct. Dyn. 4, 054101 (2017).

[19] K. Bennett, M. Kowalewski, J. R. Rouxel, and S. Mukamel, Proc. Natl. Acad. Sci. U.S.A. 115, 6538 (2018).

[20] G. Dixit and R. Santra, Phys. Rev. A 96, 053413 (2017).

[21] G. Dixit, O. Vendrell, and R. Santra, Proc. Natl. Acad. Sci. U.S.A. 109, 11636 (2012).

[22] G. Dixit and R. Santra, J. Chem. Phys. 138, 134311 (2013).

[23] M. Simmermacher, N. E. Henriksen, and K. B. Møller, Phys. Chem. Chem. Phys. 19, 19740 (2017).
[24] S. Tanaka, V. Chernyak, and S. Mukamel, Phys. Rev. A 63, 063405 (2001).

[25] N. E. Henriksen and K. B. Møller, J. Phys. Chem. B 112, 558 (2008).

[26] U. Lorenz, K. B. Møller, and N. E. Henriksen, Phys. Rev. A 81, 023422 (2010).

[27] K. B. Møller and N. E. Henriksen, Struct. Bond. 142, 185 (2012).

[28] W. Schülke, Electron Dynamics by Inelastic X-Ray Scattering, 1st ed. (Oxford Science Publications, Oxford, 2007).

[29] I. Waller and D. R. Hartree, Proc. R. Soc. A 124, 119 (1929).

[30] See Supplemental Material at http://link.aps.org/ supplemental/10.1103/PhysRevLett.122.073003 for further details on the simulations, which includes Refs. [26,31-45].

[31] B. Schmidt and U. Lorenz, Comput. Phys. Commun. 213, 223 (2017).

[32] The MathWorks, Inc., MATLAB R2016a, 2016, http://www .mathworks.com.

[33] L. Wolniewicz, J. Chem. Phys. 103, 1792 (1995).

[34] G. Staszewska and L. Wolniewicz, J. Mol. Spectrosc. 212, 208 (2002).

[35] L. Wolniewicz and G. Staszewska, J. Mol. Spectrosc. 217, 181 (2003).

[36] P. J. Mohr, B. N. Taylor, and D. B. Newell, Rev. Mod. Phys. 84, 1527 (2012).

[37] L. Wolniewicz and K. Dressler, J. Chem. Phys. 100, 444 (1994).

[38] L. Wolniewicz, J. Mol. Spectrosc. 169, 329 (1995).

[39] L. Wolniewicz and G. Staszewska, J. Mol. Spectrosc. 220, 45 (2003).

[40] H.-J. Werner, P. J. Knowles, G. Knizia, F. R. Manby, M. Schütz et al., MOLPRO, version 2015.1, a package of ab initio programs, 2015, http://www.molpro.net.

[41] T. Northey, N. Zotev, and A. Kirrander, J. Chem. Theory Comput. 10, 4911 (2014).

[42] A. M. Carrascosa and A. Kirrander, Phys. Chem. Chem. Phys. 19, 19545 (2017).

[43] M. Vacher, M. J. Bearpark, M. A. Robb, and J. P. Malhado, Phys. Rev. Lett. 118, 083001 (2017).

[44] G. A. Fiete and E. J. Heller, Phys. Rev. A 68, 022112 (2003).

[45] T. Northey, A. M. Carrascosa, S. Schäfer, and A. Kirrander, J. Chem. Phys. 145, 154304 (2016).

[46] K. Bennett, M. Kowalewski, and S. Mukamel, Phys. Rev. Lett. 119, 069301 (2017).

[47] H. Ibrahim, C. Lefebvre, A. D. Bandrauk, A. Staudte, and F. Légaré, J. Phys. B 51, 042002 (2018).

[48] Y.-W. Liu, X.-X. Mei, X. Kang, K. Yang, W.-Q. Xu, Y.-G. Peng, N. Hiraoka, K.-D. Tsuei, P.-F. Zhang, and L.-F. Zhu, Phys. Rev. A 89, 014502 (2014).

[49] L.-Q. Xu, X. Kang, Y.-G. Peng, X. Xu, Y.-W. Liu, Y. Wu, K. Yang, N. Hiraoka, K.-D. Tsuei, J.-G. Wang, and L.-F. Zhu, Phys. Rev. A 97, 032503 (2018).

[50] A. R. Bainbridge, J. Harrington, A. Kirrander, C. Cacho, E. Springate, W. A. Bryan, and R. S. Minns, New J. Phys. 17, 103013 (2015).

[51] A. M. Carrascosa, T. Northey, and A. Kirrander, Phys. Chem. Chem. Phys. 19, 7853 (2017). 
[52] U. Lorenz, K. B. Møller, and N. E. Henriksen, New J. Phys. 12, 113022 (2010).

[53] C. Arnold, O. Vendrell, and R. Santra, Phys. Rev. A 95, 033425 (2017).
[54] N. Hartmann et al., Nat. Photonics 12, 215 (2018).

[55] A. Kirrander, K. Saita, and D. V. Shalashilin, J. Chem. Theory Comput. 12, 957 (2016).

[56] http://www.archer.ac.uk. 